\title{
El valor de utilidad de los contenidos escolares. Percepciones de los estudiantes de nivel primario
}

Daiana Yamila Rigo ${ }^{1}$ y Danilo Donolo²

\section{Artículo}

Material original autorizado para la publicación en la revista Psicodebate. Facultad de Ciencias Sociales. Universidad de Palermo.

Recibido 03-03-2017 | Aceptado 17-04-2017

\section{Resumen}

El presente artículo describe el desarrollo de una experiencia didáctica con el objetivo de rescatar el valor de utilidad de los contenidos escolares y conocer la percepción de los estudiantes que participaron del diseño instructivo propuesto. La investigación se llevó a cabo con alumnos de sexto grado de nivel primario de educación con una edad promedio de 11 años. Para la recolección de datos se usaron cuestionarios y entrevistas semiestructuradas. Los resultados encontrados mostraron que la propuesta fue percibida como positiva, en tanto se rescata la tarea académica realizada como una instancia que facilita la comprensión del vínculo entre lo que se enseña en la escuela y la vida cotidiana. Las conclusiones enfatizan la importancia de lo hallado para pensar la enseñanza y el aprendizaje y la configuración de contextos educativos que apuesten a la transferencia de conocimientos y habilidades hacia la vida cotidiana.

Palabras clave: retos educativos actuales, valor de utilidad, diseño instructivo.

1 Universidad Nacional de Río Cuarto. Consejo Nacional de Investigaciones Científicas y Técnicas (CONICET). Argentina. daianarigo@hotmail.com

2 Universidad Nacional de Río Cuarto. Consejo Nacional de Investigaciones Científicas y Técnicas (CONICET). Argentina. 


\title{
The utility value of school contents. Perceptions of primary level students
}

\begin{abstract}
This article describes the development of a didactic experience with the aim of rescuing the utility value of school content and knows the perception of students who participated in the proposed instructional design. The research was conducted with students from 6th grade of primary education, with an average age of 11 years. Questionnaires and semi-structured interviews were used for data collection. The results showed that the proposal was perceived as positive, as long as you rescue the academic work carried out as an instance that facilitates the understand of the link between what is taught in school and everyday life. The findings emphasize the importance of the found to think about teaching and learning and the settings of educational contexts that are committed to the transfer of knowledge and skill into every life.
\end{abstract}

Keywords: today's educational challenges, utility value, instructive design. 
A diario, observamos distintos escenarios que nos llevan a pensar que la utilidad del conocimiento escolar muchas veces pasa desapercibido por docentes, alumnos, directivos y otros actores educativos. Una viñeta de Quino $^{i}$ es alusiva en este sentido cuando la maestra pregunta a Libertad "El sol sale por..." y ella contesta "Por la mañana (...), por la ventana del living". Situaciones similares acontecen con frecuencia en las aulas. La maestra de naturales pregunta a Mateo "¿cómo haces para saber el estado del tiempo por la mañana?". Él, con su gran astucia, sencillez y seguridad le contesta "miró por la ventana". En otra ocasión, muy parecida, en una evaluación de Ciencias Sociales, cuyo objetivo era valorar el uso del diccionario, entre las palabras que debían buscar los alumnos estaba loteo. Miguel estaba seguro del significado de la palabra, claro, al lado de su casa se estaba iniciando uno... y procedió a escribir la definición "donde la gente construye casas". En rojo, la corrección decía "tenías que buscar en el diccionario". Muchas veces las respuestas de los alumnos muestran el valor de utilidad del conocimiento, mirando por la ventana puedo apreciar el tiempo, ya sea registrando la nubosidad, el rocío, el viento, o simplemente, observando si es un día lluvioso o soleado, más allá de haber escuchado el pronóstico la noche anterior. También el conocimiento construido de manera situada es más significativo y comprensivo que la definición en abstracto que ofrece un diccionario, además Miguel tenía muy en claro que el diccionario se usa en aquellas situaciones donde se desconoce el significado de la palabra. Pensamos que estas y otras tantas experiencias que escuchamos a diario sobre las clases animan a pensar en alternativas para enseñar y aprender que consideren y den respuesta a la pregunta: ¿Para qué aprendo esto en la escuela?

En este marco, presentamos una investigación que tiene como objetivo el desarrollo e implementación de una experiencia pedagógica que busca rescatar el valor de utilidad del conocimiento escolarizado para el contexto de la vida cotidiana. De igual modo, el estudio desarrollado pretende favorecer un contexto de aprendizaje que promueva la autonomía, la curiosidad, el interés y el desafío como características que mejoran el compromiso del alumno hacia las tareas académicas llevadas a cabo en la escuela. El diseño se llevó a cabo con estudiantes y docentes de educación primaria de sexto grado, cuyas edades se distribuyen en un rango de 10 a 12 años, en torno al eje curricular Espacios Geográficos.

Entendemos que los desarrollos teóricos y empíricos que puedan derivarse de la presente investigación atienden a un doble reto. Primero, la importancia de investigar in situ, junto a docente y alumnos, en un aula de educación primaria 
donde acontecen infinidad de situaciones de enseñanza y aprendizaje. Segundo, comprender y avanzar en nuevas propuestas educativas que, pensadas para rescatar el valor de utilidad del conocimiento, generen un clima de aprendizaje que promueva mayor interés, motivación intrínseca y compromiso cognitivo, afectivo y conductual.

\section{Ir a la escuela...enseñar y aprender frente a los retos actuales}

Hace algo más de tres décadas, Gardner (1983) se pronunciaba con una nueva idea sobre inteligencia que tuvo grandes repercusiones en el campo educativo. Idea que luego en el libro La mente no escolarizada (Gardner, 2003) retomó para enfatizar que el aprendizaje debe conducir a la creatividad, curiosidad, búsqueda de nuevas ideas, resolución de problemas reales, imaginación y al trabajo colaborativo como pilares fundamentales de la educación. De esta manera, alentaba a los docentes a repensar el propósito y el funcionamiento de las escuelas, a través de la implementación de nuevas estrategias y recursos educativos que permitan redefinir qué es el aprendizaje y cómo sucede.

La pregunta que direcciona esta meta quedaría expresada de la siguiente forma: ¿Qué pasaría si intentáramos desarrollar una nueva cultura de pensamiento en nuestras escuelas, aulas, museos, universidades, entre otros? De manera tal de transformar los colegios en espacios que promuevan aún más la autonomía, la curiosidad, las múltiples inteligencias y el compromiso. Intentar este cambio exige averiguar qué tipo de pensamiento debemos promover en los contextos educativos.

Al respecto, Ritchhart $(2002,2012)$ hace mención a que el entorno de aprendizaje debe pensarse como un espacio para cultivar el descubrimiento, la emoción y el disfrute en el acto de pensar, a la vez de proporcionar a los estudiantes cierto grado de autonomía, independencia y elección junto a estructuras que faciliten interacciones positivas en torno a grandes ideas u objetos. Se interroga: ¿Qué tipos de pensamiento son de valor? En términos generales aspiramos a que los estudiantes puedan usar distintos tipos de pensamientos: formular preguntas, establecer conexiones entre ideas y disciplinas diversas, explorar objetos culturales desde diferentes perspectivas y puntos de vistas alternativos, observar con detalle para reconocer matices y profundizar la comprensión, e identificar y buscar evidencias para justificar argumentos y explicaciones.

En una comunicación anterior, Ritchhart y Perkins (2000) ponen el acento en cómo crear un lugar donde el pensamiento sea valorado, visible y activamente promovido, donde se propicie la trasferencia de habilidades y conocimientos a nuevos contextos, donde se desarrolle la comprensión profunda, la motivación, el compromiso y el desarrollo de la autonomía. Acuerdan en tres condiciones instruccionales para el desarrollo del aprendizaje pleno: indagar sobre nuevas 
experiencias, ideas e intereses; explorar posibilidades y perspectivas novedosas como ocasiones para ir más allá del dato, e introducir la ambigüedad en la presentación del conocimiento como provisorio más que absoluto.

Krajcik y Blumenfeld (2006), en esta discusión sobre qué pensamiento cultivar, entienden que promover el valor de aprender a partir de la formulación de preguntas es un recurso interesante en el enfoque de aprendizaje por proyectos. Los autores ponen el acento en el aprender investigando, haciendo lo mismo que los científicos realizan cuando trabajan en sus disciplinas. Proponen, entre otras características, dos que permiten explorar diversos fenómenos, buscar respuestas alternativas, discutir ideas, cambiar visiones y experimentar nuevas ideas: las preguntas mentoras y las experiencias de anclaje. La primera alude a preguntas para organizar y conducir el pensamiento, en tanto proveen un contexto en el cual los alumnos pueden usar y explorar metas de aprendizaje, prácticas científicas y proporcionan continuidad y coherencia a la tarea de aprendizaje propuesta. La segunda, refiere a experiencias significativas y relevantes que ayudan a relacionar las nuevas ideas y objetos en un contexto significativo para comprender la importancia del tópico (ejemplos: videos, relatos, narrativas, entro otros recursos).

La clave de las preguntas en la secuencia didáctica para estimular la curiosidad y la creatividad ha sido un tema investigado por Csikszentmihalyi (1996) y retomado por Rinaudo y Donolo (2013), quienes insisten en que las preguntas son una fuente inagotable de ideas y de ocurrencias fructíferas. Aun aquellas más complejas y hasta absurdas o mal formuladas y de contenido vago son motivo de atención, promueven la imaginación, inspiran el pensamiento y generan acción. Asimismo, plantean la importancia de brindar la oportunidad para plantear preguntas, formular cuestiones y expresar puntos de vistas alternativos como rasgos contextuales que promueven el aprendizaje autónomo, la motivación y el compromiso.

Otros rasgo que promueve el interés y el aprendizaje pleno es la autonomía, o la oferta de opciones y su aprovechamiento. En general, estamos acostumbrados a recibir recetas, pero, parece ser que tener y contar con la posibilidad de elegir qué y cómo aprender es una buena manera de alimentar el entusiasmo. Perkins (2010) explica que el aula envuelve muchas veces una importante cuota de restricción -qué se debe dominar, cuándo se deben hacer las cosas, qué protocolos de conductas observar -, y aclara que, si bien es necesaria una cierta estructura, también es buena la flexibilidad, no sólo en las opciones, sino también en el modo de presentar el conocimiento y las ideas.

Se trata de propuestas e ideas educativas que tienen como meta crear oportunidades para pensar y hacer visible el pensamiento que ofrecen espacios para pensar con, más que pensar acerca de, para revisar, reflexionar conceptos o comportamientos de la vida cotidiana. Ritchhart, Church y Morrison (2014), 
proponen prácticas de enseñanza que no tengan como fin entregar información, sino actividades donde los estudiantes puedan comprender e involucrarse con las ideas a estudiar. Por eso consideran crucial la configuración del contexto instructivo para favorecer alumnos implicados con los contenidos, en tanto encuentren sentido para su estudio y discusión: "cuando hay algo importante que vale la pena pensar y existe una razón para pensarlo en profundidad, nuestros estudiantes experimentan el tipo de aprendizaje que tiene un impacto duradero y una influencia poderosa, no sólo a corto plazo sino también con el transcurrir del tiempo" (Ritchhart et al., 2014, p. 63).

Asimismo, para lograr involucrar al estudiante en el estudio de ideas complejas, no sólo el sujeto tiene un rol importante, sino sobre todo la configuración del contexto instructivo. Al respecto, distintos aportes enfatizan el interjuego sujetocontexto. El modelo contextual sobre compromiso -cognitivo, conductual y afectivo- de Lam, Wong, Yang y Liu (2012) es elocuente en su propuesta al integrar los factores contextuales, personales y sociales junto al rendimiento para explicar y comprender la implicación de los alumnos. Entre los rasgos del contexto identifican seis componentes importantes: el desafío, la autenticidad, la curiosidad, la autonomía, el reconocimiento y la evaluación. Al referirse a los rasgos personales, por un lado se destacan las creencias motivacionales orientación a metas, autoeficacia y atribuciones- y por otro lado, el compromiso afectivo, conductual y cognitivo del estudiante. Y se remarca la importancia sobre cómo se percibe el entorno instructivo, en tanto es comprendido como soporte para el desarrollo de la implicación y la motivación.

En la misma línea, Darr (2012), Fredriscks, Blumenfeld y Paris (2004), Hipkins (2012), Reschly y Christenson (2012), Rigo (2013), Rigo y Donolo (2014a) y Shernoff (2013), acuerdan que el compromiso debe definirse en función de la interacción entre una persona y un entorno de aprendizaje. Asimismo, coinciden en pensar al compromiso como un constructo complejo que integra no sólo la parte cognitiva, sino también lo afectivo y lo conductual. Estos estudios encuentran que los factores del entorno hacen la diferencia entre un alto o bajo compromiso más que los factores personales. Entre los primeros se remarca la importancia de que el contexto sea percibido como auténtico y desafiante, con metas claras y con valor de utilidad. Entre los segundos se resaltan el participar activamente, formular preguntas, solicitar aclaraciones, iniciar debates, colaborar con pares, así como el nivel de habilidad y el nivel de logro.

Como observamos en este breve recorrido, los diversos desarrollos teóricos con fundamento en la práctica aportan ideas distintas pero complementarias para cultivar un nuevo tipo de pensamiento en la enseñanza, ofrecer distintas alternativas y orientaciones para promover el aprendizaje autónomo, la curiosidad y el compromiso 
de los estudiantes. Estamos convencidos de que definir contextos educativos con los rasgos antes descriptos nos permitirá no sólo contar con nuevas contribuciones teóricas que posibiliten ampliar los desarrollos inscriptos en la Psicología Educacional, sino también generar la formación de recursos -docentes, alumnos e investigadorespropicios para definir contextos alternativos para aprender y pensar.

\section{Método}

\section{¿Qué nos propusimos?}

Desarrollar una tarea académica para rescatar el valor de utilidad de los contenidos escolares en el área de las Ciencias Sociales, promover la transferencia hacia la vida cotidiana, así como evaluar la percepción de los estudiantes sobre la experiencia desarrollada.

\section{La experiencia de investigación ¿Con quiénes?}

El diseño de la actividad se llevó a cabo junto a la docente de sexo grado del área de Ciencias Sociales. De la experiencia participaron 23 alumnos que estaban cursando el último año de la educación primaria obligatoria, con una media de 11 años. La investigación se desarrolló en un Centro Educativo Público de la localidad de Las Higueras, provincia de Córdoba, luego de solicitar el consentimiento informado a directivos, padres, alumnos y docente. La investigación se enmarcó dentro de la metodología estudio de diseño (Rinaudo \& Donolo, 2010), en tanto se tienen como objetivo profundizar en lineamientos teóricos que ayuden a su vez a mejorar las prácticas de enseñanza y aprendizaje.

\section{La tarea académica ¡Y si damos la vuelta al mundo!}

La tarea académica diseñada proponía a los estudiantes que investigaran, en parejas, sobre una ciudad, de libre elección, para orientar el viaje de una adolescente que acababa de cumplir 15 años, a quien debían escribirle una carta para compartir la información encontrada. El objetivo pedagógico perseguido fue que los estudiantes conocieran distintas herramientas y recursos para acceder a nuevos conocimientos, saberes e información, y profundizar a la vez en la comprensión de conceptos centrales del eje curricular Espacios Geográficos y promover la utilización de los contenidos enseñados para pensar una situación real.

En primera instancia, se les propuso que exploraran el globo terráqueo y eligieran un continente, un país y una ciudad en función de sus intereses y gustos por conocer y explorar. Se los incitaba a: "Mirar, leer, tocar, consultar, intercambiar, preguntar y señalar en el globo terráqueo un lugar preferido del mundo a descubrir..."

En segunda instancia, los estudiantes fueron invitados a averiguar la distancia 
que había desde Las Higueras al lugar de destino elegido. La actividad se desarrolló en la sala de computación. Cada alumno calculó la cantidad de kilómetros a recorrer, que luego representaron en un planisferio, haciendo uso de distintas aplicaciones de la Web. Algunas preguntas que orientaron la búsqueda fueron:

¿Qué tan lejos o cerca queda?, ¿cuántas horas, días o meses tardaríamos en llegar?, ¿qué transporte tendremos que tomar, un auto, un colectivo, un avión, un barco, una bici o simplemente caminado?, ¿tenemos sólo una opción o varias alternativas -rutas- para llegar a ese lugar? y ¿cuál preferirías y por qué?

En tercera instancia, se les presentó la consigna de la actividad ofreciendo el siguiente texto:

Constanza, una adolescente de 15 años de edad, quiere como regalo para su cumpleaños viajar a ese lugar que tú elegiste. No sabe mucho. Bueno, casi nada y necesita de tu guía. El viaje se programa para realizarse a mediados de agosto. Necesitará que le brindes mucha información para que pueda armar su maleta, programar visitas, probar comidas típicas, aprender de las costumbres y hábitos de la gente del lugar, hasta quizás deba tomar un curso de idioma antes de partir para poder comunicarse allá. También tendrá que saber sobre el cambio horario para comunicarse con su familia y por lo menos contar con un pequeño plano para orientarse. Podrías averiguar toda esa información y escribirle una carta para orientarla, y así pueda disfrutar de su corta estadía en

En cuarta instancia, para iniciar la búsqueda de información previa a la escritura de la carta, como experiencia disparadora se invitó a una joven viajera para que relatara sus diversas experiencias por el mundo, aquello que fue aprendiendo de sus viajes, los detalles a tener presentes, cómo armar la maleta para cada destino, qué tener presente para organizar un viaje, los problemas in situ y cómo solucionarlos, entre otros aspectos.

En quinta instancia, se animó a los estudiantes a formular preguntas para orientar la búsqueda de información, tanto en la biblioteca como en la sala de computación con acceso a Internet, para empezar a escribir un primer esbozo de la carta. Cabe destacar que cada alumno contaba con un borrador, donde iba registrando los resultados de las búsquedas, preguntas y consultas, de modo de ir apuntando los avances que producían hacia la redacción de la carta final y resumiendo la información recolectada.

La actividad se desarrolló en el marco del eje curricular Espacios Geográficos propuestos dentro de los Núcleos de Aprendizajes Prioritarios (NAP) para la enseñanza de sexto grado. Los contenidos centrales que se trabajaron durante 
la experiencia refieren a las distintas formas de representar la tierra, los continentes, las tres américas, los movimientos de la tierra -rotación, traslación-: las estaciones, el día y la noche, líneas imaginerías: ecuador y meridiano de Greenwich, hemisferio oriental vs. occidental, tipos de clima, husos horarios, escala gráfica y numérica en mapas, el género epistolar y sus partes.

Algunas de las preguntas realizadas por los alumnos durante la experiencia y que fueron disparadoras para trabajar los diversos conceptos del eje de interés, fueron:

- ¿Por qué en agosto en Europa es verano y en América del Sur es invierno?

- ¿Por qué hay diferencia horaria entre Las Higueras y los diversos destinos propuestos para el viaje de Constanza?

- ¿Cómo se explica el tipo de clima de cada región? ¿Por qué algunos lugares son cálidos o tropicales todo el año?

- ¿Qué herramientas puedo usar para calcular la distancia desde Las Higueras al lugar de destino?, ¿sólo Google Maps o Google Earth?, ¿y si no anda Internet?

Cada contenido se trabajó con el grupo de alumnos a medida que iban surgiendo dudas, consultas e interrogantes como los mencionados con anterioridad.

Al plantear la tarea académica se contempló que la misma tuviera presente los siguientes rasgos teóricos, a saber: autonomía de los estudiantes en la elección de la ciudad, al ofrecer la posibilidad de elegir el lugar destino del viaje de la adolescente, que a la vez promoviera la curiosidad y el interés por saber; autenticidad, en tanto la situación de aprendizaje se encuentra relacionada con una experiencia vinculada a un hecho realizable en la vida cotidiana, habilitando una instancia para facilitar la transferencia de habilidades y conocimientos; novedad y diversidad de recursos, en un planteo original e inesperado de la tarea de investigación, que habilitaba el uso de diversos recursos, fuentes de información y perspectivas y, a la vez, un espacio para la formulación de preguntas; y desafiante, formulada dentro de la zona de desarrollo próximo de los estudiantes, una propuesta que los animaba a ir más allá de los conocimientos que tenían, pero brindando soportes para avanzar hacia la meta propuesta y nuevos aprendizajes.

\section{Los datos e instrumentos}

Para conocer las percepciones de los estudiantes sobre la experiencia de aprendizaje desarrollada se utilizó un cuestionario con seis preguntas cerradas de opción múltiple (ver anexo A), que consultaban sobre el valor de utilidad de la tarea, las posibilidades que ofrecía la actividad para promover el interés, la curiosidad, la autonomía, el disfrute y atribuir importancia a los aprendizaje logrados; y una entrevista semi-estructurada, para profundizar en las respuestas ofrecidas por los estudiantes, de modo de ampliar sus valoraciones hacia la experiencia de 
aprendizaje. Asimismo, para profundizar el análisis y la interpretación de los datos se realizaron observaciones antes del desarrollo de la experiencia.

\section{Análisis y discusión de resultados}

Entre los resultados principales encontramos que los estudiantes tienden a valorar, en general, como positiva la experiencia desarrollada. Un 83\% informan que la tarea permitió establecer vínculos con situaciones de la vida cotidiana, reflexionado sobre problemas reales y usando el conocimiento aprendido en Ciencias Sociales para solucionar un problema real. Los estudiantes comparten que la experiencia fue muy interesante; en un $70 \%$, y el 30\% restante marcó que fue interesante o algo interesante. Respecto a la dificultad de la tarea, los estudiantes mostraron opiniones más variadas, tan solo un 57\% la percibieron en un nivel intermedio, ni fácil ni difícil, y el 15\% de los estudiantes la percibió como un poco simple y el 28\% como un poco difícil. Del total de los estudiantes, el 61\% le pareció muy interesante poder elegir la ciudad a investigar, y el resto optó por la alternativa de respuesta interesante. Las actividades que más implicaron fueron la búsqueda de información tanto en Internet como en la biblioteca reportado por el 70\% de los estudiantes, en segundo orden las explicaciones de la docente con un 65\%. Siguen la escritura de la carta y las visitas con un 49\% de elección, y ocupan los porcentajes más bajos de preferencias las tareas orientadas a contestar preguntas y leer el libro de ciencias. En general, los que participaron de la experiencia reportan en un $61 \%$ que la tarea les permitió comprender la importancia de aprender Ciencias Sociales, un $30 \%$ lo consideró como muy relevante y tan solo un $9 \%$ la percibió como aburrida.

Son igualmente interesantes las justificaciones y explicaciones que ofrecen los estudiantes sobre la experiencia, resaltando aspectos que se facilitaron y condujeron a nuevos aprendizajes.

Los estudiantes tuvieron la posibilidad de desarrollar nuevos aprendizajes a lo largo de la experiencia. Un estudiante expresa: "La investigación me enseñó muchas cosas sobre ese lugar, también cómo buscar en un libro...nosotras no sabíamos buscar en el índice. Siempre nos dicen la página donde está la información”. Este comentario informa sobre la importancia de ofrecer instancias para desarrollar competencias específicas. Pareciera que dar lugar a nuevos aprendizaje está asociado a experiencias libres y con mayor autonomía en la toma de decisiones. Aunque parezca fácil, saber buscar en un índice primero requiere tener en claro qué información se desea encontrar en él, luego elegir palabras clave para iniciar la búsqueda y explorar el índice, tener una idea clara hacia dónde se va y qué se desea hallar. Las prácticas escolares, como lo mencionan los alumnos, a veces restringen esas oportunidades de apertura a nuevos desafíos, al ofrecer a modo de delivery indicaciones que no 
habilitan alternativas de resolución. En las observaciones de clase realizadas y en los registros tomados desde el pizarrón, se plasma lo expresado por los estudiantes; las consignas siempre indican en qué libro buscar y en qué página.

- Trabajamos con el libro de Biciencia Página 194. Observamos el mapa de Argentina en el que se muestra cómo era el país entre 1852 y 1861.

- Leemos y analizamos la página 195, 196, 197 y 198 para luego responder...

- Trabajamos la pág. 262 del libro.

En los comentarios de los estudiantes también se cuela que el aprendizaje deja de ser lineal, en tanto no responde al orden prescripto por el libro de ciencias, sino que se produce a la par de los avances de los estudiantes en la investigación, cuando surgen interrogantes, dudas e inquietudes. Al respecto, el estudio de Harris (2010) concluye que los docentes que entregan el conocimiento, más que ofrecer posibilidad para su co-construcción, son aquellos que promoverían menos la implicación de los estudiantes y sus intereses. Por eso, animamos a usar la biblioteca como un espacio educativo que favorezca el aprendizaje autónomo (Marzal, Díaz \& Calzada, 2012).

Comentarios similares surgen sobre el uso de internet. Uno de los grupos expresa con claridad su preocupación sobre la validez y lo fiable que es la información que está ahí, en la nube: "Nosotros encontramos información distinta, no era igual, cada página decía algo distinto, no sabíamos cómo hacer para saber qué estaba bien o mal". Responder a estas inquietudes supone atender al desarrollo de una alfabetización informacional, definida por la UNESCO (2011) como la capacidad de las personas y los grupos de reconocer necesidades de información, localizar y evaluar la calidad de la misma, almacenar y recuperar datos, aplicar la información para crear y comunicar conocimiento de manera eficaz y ética. No basta con tener acceso a una computadora con Internet, se requiere asimismo cuestionar las fuentes de los datos y reconocer los contextos culturales y sociales dentro de los cuales la información fue creada para guiar la selección de la información y evaluar su pertenencia y veracidad. Así, la experiencia desarrollada animó a docentes y a estudiantes a generar estrategias educativas para evaluar la vertiginosa información que fluye por internet, competencias necesarias para comprender y buscar entre la diversidad de fuentes disponibles. Otro grupo pone énfasis en la importancia de aprender a buscar y seleccionar información: "aprender cómo buscar información, reflexionar cada cosa, antes de guardar la página...tuvimos que aprender a distinguir entre buena y mala información". Al respecto, Wales (2015), fundador de Wikipedia, apunta que el analfabetismo del futuro es no saber utilizar bien Internet, destacando que la educación actual tiene que estar dirigida a formar un alumno competente en el manejo de la información. 
Otros de los aspectos resaltados de la experiencia fue la visita de la joven viajera. Un alumno dice: "Nos ayudó a ver lo importante de aprender Ciencias Sociales...aprender algo importante, para planificar mis vacaciones, y escribir una carta para guiar el viaje con lo que aprendemos acá". Su comentario refleja el valor de utilidad de los contenidos escolares cuando se ofrecen experiencias educativas que brindan andamios para posibilitar la transferencia de lo aprendido a contextos cotidianos. Se destacan dos aspectos centrales. Primero, la importancia de que la escuela enseñe contendidos útiles y sirvan a futuro para tomar decisiones. Perkins (2010) expone que la escuela no debe preparar a los estudiantes sólo para rendir bien un examen, debe, asimismo, planificar una enseñanza que establezca vínculos concretos entre los contenidos y la vida cotidiana. Y segundo, la necesidad de promover situaciones de transferencia para que los estudiantes puedan establecer relaciones entre la teoría y la práctica, tal que el docente trabaje junto al estudiante para guiar el aprendizaje.

Los estudiantes también rescataron el valor del aprendizaje contextualizado: "Utilizar la información cuando la necesitamos, no aprender así -toma un libro en sus manos y empieza a pasar las hojas-. Escribir la carta fue un desafío, no podíamos poner señora, era una adolescente, tampoco tuvimos que poner toda la información que encontramos en el libro o en internet, teníamos qué decidir qué era importante para guiar". En estos comentarios no sólo se transmite la importancia de aprender con una finalidad y un objetivo claro, leer para buscar información, y no simplemente por leer; y escribir para un destinatario, no para contestar preguntas. Los alumnos marcan la importancia de delimitar con claridad los propósitos de las tareas académicas, en tanto ese marco facilita la toma de decisiones: "saber qué información podía ser importante para la adolescente y escribir una carta que le ayude". En este sentido, Mitchell y Carbone (2011) mencionan que las tareas auténticas y con propósitos bien definidos, ofrecen la posibilidades de ir chequeando los progresos, reflexionando sobre el proceso de aprendizaje, ajustando y modificando las producciones para lograr la meta de aprendizaje.

Las tareas observadas y registradas antes de llevar a cabo la tarea de investigación muestran que son simples ejercicios de preguntas y respuestas, que se encuentran de manera ordenada y casi textual en las páginas del libro de ciencias indicadas por la docente. Una práctica que no se alinea a la ida de transferencia y aprendizaje contextualizado, sino más bien a una acción de copie y pegue (Rigo \& Donolo, 2016).

(Dictado-Deber)

Leemos y analizamos las páginas 195, 196, 197 y 198 para luego responder:

1. ¿Quiénes firmaron el Acuerdo de San Nicolás?

2. ¿Qué objetivos perseguía este acuerdo? 
3. ¿Por qué la Provincia de Buenos Aires decidió separarse de la Confederación Argentina?

4. ¿Qué medidas tomó Buenos Aires cuando se separó de la Confederación?

5. ¿Qué derechos establecía la Constitución de 1853 ?

6. ¿Cuál es la forma de Gobierno adoptada por la Confederación? Explica

7. El Pacto de San José de Flores ¿acordó la incorporación de Buenos Aires a la Confederación? Justifica.

8. La Ley de Derechos Diferenciales sancionada por Urquiza ¿Tuvo como resultado el crecimiento del comercio internacional en la Confederación? ¿Por qué?

(Copia pizarrón)

Revisamos, corregimos y completamos la tarea de la clase anterior

-La docente pregunta ¿pudieron hacer la tarea?

-Un alumno contesta: Sí, leíamos y ahí estaban.

-Otro alumno expresa: fue fácil, leía y estaba todo en el libro.

Al respecto, Furman (2015), remarca la importancia de unir este tipo de prácticas, que no son más que preguntas fácticas, con preguntas para pensar; situaciones problemáticas que impliquen a los estudiantes cognitivamente en el desafío de aprender haciendo, buscando e investigando. Y ofrecer así espacios educativos que enseñen a pensar y usar el conocimiento.

Por último, los estudiantes expresan su implicación en la tarea, como otro de los aspectos positivos valorados: "Con este trabajo me quede impresionada, aprendí muchas cosas que no sabía que ni existían. Lo que más me gustó fue cuando empezamos a buscar en el globo terráqueo el lugar sobre el que íbamos a buscar la información. Para hacer la tarea nos sirvió la ayuda de muchos, la seño y de nuestros compañeros, los encuentros, las notas en nuestro borrador, las preguntas que formulamos". Como remarcan Hospel y Galand (2016), no sólo es importante promover la autonomía sino también ofrecer una buena estructura para comprometer al estudiante en la tarea. La libertad de elección ofrece el puntapié inicial para motivar e interesar al estudiantes en sus aprendizajes, estudiar algo placentero y cautivador, favoreciendo mayores niveles de compromiso afectivo; pero el soporte que el docente ofrece en el camino es igual de importante cuando se ajusta al nivel de la tarea y se encuentra dentro de la zona de desarrollo próximo de los estudiantes, promoviendo el compromiso conductual y cognitivo (Rigo \& Donolo, 2014b). Como se refleja en los comentarios de los alumnos, ambos aspectos son importantes para atender a la experiencia desarrollada y muestran cuán relevante fueron para sus aprendizajes y niveles de implicación.

Sin lugar a dudas, estos comentarios explican los porcentajes iniciales 
encontrados; realmente los estudiantes que participaron de esta investigación tuvieron la posibilidad de salir de la rutina, que dice más o menos qué se debe hacer, cómo y a qué ritmo, y trazaron nuevos caminos y senderos en sus experiencias de aprendizaje, generaron nuevas habilidades para buscar información con recursos educativos tradicionales, como los libros, y recursos educativos digitales. Asimismo, se implicaron en la tarea al tener la posibilidad de elegir, a la vez contar con el soporte del docente, quien ayuda a establecer los primeros nexos entre lo que se aprende y la vida fuera del aula.

\section{Seguir pensado la educación...}

A modo de consideración final, la presente investigación tuvo como objetivo conocer y describir las perspectivas que un grupo de estudiantes construyó en torno a una tarea académica planificada para promover la autonomía, la curiosidad, el interés, el desafío y sobre todo el valor de utilidad de lo aprendido. Entre los resultados encontramos una valoración positiva de la experiencia desarrollada. Los sujetos, en su gran mayoría, indican que la realización de la actividad fue interesante para pensar vínculos entre los contenidos y la vida cotidiana, e igualmente manifiestan su implicación en el desarrollo de la propuesta, mostrando afinidad por la misma como modalidad de enseñanza y aprendizaje.

Asimismo, lo hallado nos motiva a seguir pensando en tareas académicas que valoren el conocimiento que se aprende dentro del aula, creando espacios educativos que no sólo busquen aplicar lo que se aprende a su contexto original, sino también a un entorno mucho más lejano, apreciando su valor de utilidad para metas futuras y promoviendo acciones de transferencia. De igual modo, la investigación desarrollada y los resultados derivados nos permiten trazar una agenda de metas educativas, como la necesaria alfabetización informacional, sin olvidar rescatar los contextos más tradicionales de enseñanza y de aprendizaje, como lo es la biblioteca escolar y la exploración de libros. Consideramos que ambos recursos son complementarios y fundamentales.

También destacamos el diseño de la tarea académica, y rescatamos los rasgos de autonomía y el soporte ofrecido a lo largo de la misma, características que los estudiantes marcan como positivas a la hora de evaluar su implicación en la experiencia. Factores que contribuyeron a generar un contexto de aprendizaje que motiva la indagación y nuevas búsquedas, no pautadas de antemano, pero con una estructura didáctica que posibilita avances en el desarrollo y logro de nuevos conocimientos.

A partir de lo encontrado, consideramos importante generar y diseñar contextos educativos en el nivel primario de educación que ofrezcan a los estudiantes 
posibilidades de aprender a partir de procedimientos alternativos y originales tanto para gestionar la información, como para otorgar valor de utilidad al contenido escolar. Creemos que se trata de una experiencia innovadora que muestra una forma de hacer diferente en pro de mejorar los aprendizajes de los estudiantes, y de promover el compromiso de éstos, fomentando la curiosidad, la toma de decisiones, la imaginación, la búsqueda de información, la formulación de preguntas y el planteo de ideas para resolver problemas reales sin recetas, ni caminos prefijados.

i Una versión de la viñeta de Quino se puede consultar en https://salinero2013.files.wordpress. com/2013/05/quinosol.png

\section{Referencias}

Csikszentmihalyi, M. (1998). Creatividad. El fluir y la psicología del descubrimiento y la invención. Barcelona: Paidós.

Darr, C. (2012). Measuring student engagement: the development of a scale for formative use. En S. Christenson, A. Reschly, \& C. Wylie (Eds.), Handbook Research on Student Engagement (pp. 707-724). Minneapolis, MN: Springer. https://doi.org/10.1007/978-1-4614-2018-7_34

Fredriscks, J. A., Blumenfeld, P. C., \& Paris, A. H. (2004). School engagement: Potencial of the concept state of the evidence. Review of Educational Research, 74(1), 59-109. https://doi.org/10.3102/00346543074001059

Furman, M. (2015). Preguntas para pensar. Conferencia TEDxRíodelaPlataED. Recuperado de http://www.tedxriodelaplata.org/videos/preguntas-para-pensar

Gardner, H. (1983). Frames of Mind, The Theory of Multiple Intelligences. New York: Basic Book.

Gardner, H. (2003). Inteligencias múltiples. La teoría en la práctica. Buenos Aires: Paidós.

Harris, L. (2010). Delivering, Modifying or Collaborating? Examining Three Teacher Conceptions of How to Facilitate Student Engagement. Teachers and Teaching, 16(1),131-151. https://doi.org/10.1080/13540600903478037

Hipkins, R. (2012). The engaging nature of teaching for competency development. En S. Christenson, A. Reschly, \& C. Wylie (Eds.), Handbook Research on Student Engagement (pp. 441-456). Minneapolis, MN: Springer. https://doi. 
org/10.1007/978-1-4614-2018-7_21

Hospel, V. \& Galand, B. (2016). Are both classroom autonomy support and structure equally important for students' engagement? A multilevel analysis. Learning and Instruction, 41, 1-10. https://doi.org/10.1016/j. learninstruc.2015.09.001

Krajcik, J. \& Blumenfeld, P. (2006). Project-Based Learning. En K. Sawyer, (Ed.) The Cambridge Handbook of the learning Sciences (pp. 317-333). New York: Cambridge University Press.

Lam, S. F., Wong, B., Yang, H., \& Liu, Y. (2012). Understanding Student Engagement with a contextual Model. En S. Christenson, A. Reschly, \& C. Wylie (Eds.) Handbook Research on Student Engagement (pp. 403-420). Minneapolis, MN: Springer. https://doi.org/10.1007/978-1-4614-2018-7_19

Marzal, M., Díaz, M. \& Calzada, J. (2012). Un modelo y un método para la transformación de la biblioteca escolar en centro de recursos de enseñanza y aprendizaje. TransInformação, 24(3), 165-178. https://doi.org/10.1590/S010337862012000300002

Mitchell, I. \& Carbone, A. (2011). A typology of task characteristics and their effects on student engagement, International Journal of Educational Research, 50(5-6): 257-270. https://doi.org/10.1016/j.ijer.2011.05.001

Perkins, D. (2010). El aprendizaje pleno. Principios de la enseñanza para transformar la educación. Buenos Aires: Paidós.

Reschly, A. \& Christenson, S. (2012). Jingle, Jangle, and Conceptual Haziness: evolutions and future directions of the engagement construct. En S. Christenson, A. Reschly, \& C. Wylie (Eds.). Handbook Research on Student Engagement (pp. 3-20). Minneapolis, MN: Springer. https://doi. org/10.1007/978-1-4614-2018-7_1

Rigo, D. (2013). Compromiso hacia las tareas académicas. Diseños instructivos e inteligencias múltiples. Ikastorratza, e-Revista Didáctica, 10.

Rinaudo, C. \& Donolo, D. (2010). Estudios de diseño. Una alternativa promisoria en la investigación educativa. RED - Revista de Educación a Distancia, 22.

Rinaudo, C. \& Donolo, D. (2013). Inteligencia-Creatividad (y la escuela). En D. Donolo \& R. Elisondo (Eds.), Estudio de creatividad Las travesias de 
Alfonsina, de Astor, de Julios y de Marías (pp. 17-46). España: Sociedad Latina de Comunicación Social.

Rigo, D. \& Donolo, D. (2014a). Entre pupitres y pizarrones. Retos en educación primaria: el aprendizaje con compromiso. Educatio Siglo XXI, 32(2), 59-80. https://doi.org/10.6018/j/202161

Rigo, D. \& Donolo, D. (2014b, noviembre). Factores clave en la promoción del compromiso en educación primaria. Un estudio sobre tasks, teachers and students. Trabajo presentado en el Congreso Iberoamericano de Ciencia, Tecnología, innovación y Educación, Buenos Aires, Argentina.

Rigo, D. \& Donolo, D. (2016). La evaluación...más de lo mismo. Desafiando formatos y modalidades sin libreto. Panorama. Revista de Ciencias Sociales, 10(19), 5-23.

Ritchhart, R. (2002). Intellectual Character. What it is, Why it matters and How to get it. San Francisco, CA: Jossey-Bass.

Ritchhart, R. (2012). The Purpose and Promise of Schools. En R. Ritchhart (Ed.). Creating Cultures of Thinking: The 8 Forces We Must Master to Truly Transform Our Schools (pp. 13-36). New York: Wiley

Ritchhart, R., Church, M. \& Morrison, K. (2014). Hacer visible el pensamiento. Cómo promover el compromiso, la comprensión y la autonomía de los estudiantes. Buenos Aires: Paidós.

Ritchhart, R. \& Perkins, D. (2000). Life in the Mindful Classroom: Nurturing the Disposition of Mindfulness. Journal of Social Issues, 56(1), 27-47. https://doi. org/10.1111/0022-4537.00150

Shernoff, D. (2013). Optimal learning. Environment to promote student engagement. New York: Springer. https://doi.org/10.1007/978-1-4614-7089-2

UNESCO (2011). Alfabetización mediática e informacional. Recuperado de http://unesdoc.unesco.org/images/0021/002160/216099S.pdf

Wales, J. (2015, 23 de octubre). 'El nuevo analfabetismo no es no saber cosas, es no saber usar la información'. El Mundo, Sección Cultura. Recuperado de http://www.elmundo.es/cultura/2015/10/23/5629fed346163f79308b45c0.html 


\section{Apéndice A}

\section{Cuestionario}

\section{Valoración de la experiencia "Y si damos la vuelta al mundo"}

Le solicitamos que responda a las preguntas brindando la información que se requiere en cada una y atendiendo a la indicación ofrecida para cada instancia.

1. La tarea realizada le permitió reflexionar sobre situaciones cotidianas y usar los conceptos centrales de las Ciencias Sociales para solucionar problemas reales (elija solo $\underline{\text { UNA }}$ opción): Sí - No

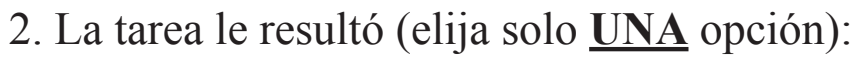
a. Muy interesante
b. Interesante
c. Algo interesante
d. Muy poco interesante
e. Nada interesante

3. La tarea le resultó (elija solo UNA opción):
a. Muy fácil
b. Muy difícil
c. Algo fácil
d. Algo difícil
e. No difícil, ni fácil, de nivel intermedio

4. ¿Qué le pareció la posibilidad de elegir la ciudad a partir de la cual iniciar la investigación? (elija solo UNA opción):
a. Muy interesante
b. Interesante
c. Algo interesante
d. Muy poco interesante
e. Nada interesante 
5. Si pudiera elegir entre varias opciones sobre cómo aprender Ciencias Sociales ¿Qué preferiría? (Elija TODAS las opciones que desee, marcando un ORDEN de preferencia):
a. Contestar preguntas por escrito
b. Escribir una carta
c. Escuchar las explicaciones de la maestra
d. Buscar información en la biblioteca y en internet
e. Leer el libro de Ciencias Sociales
f. Escuchar a invitados

6. En general, encuentra que lo aprendido durante la experiencia le fue (Elija solo UNA opción):
a. Muy aburrido
b. Aburrido
c. Algo aburrido
d. Interesante
e. Muy interesante 